\title{
Breadboard Model of On-orbit Calibration Equipment for Small Hyperspectral Sensor
}

\author{
By Yoshihide AOYANAGI ${ }^{1)}$, Shin SATORI ${ }^{1)}$ and Yusuke TAKEUCHI ${ }^{2)}$ \\ ${ }^{1)}$ Hokkaido Institute of Technology, Sapporo, Japan \\ ${ }^{2)}$ Hokkaido Satellite, Inc., Sapporo, Japan
}

(Received July 16th, 2009)

\begin{abstract}
This paper examined the effect of atmospheric correction on that hyperspectral sensors have wavelength error. Also we examined the effects of classification result on erroneous atmospheric correction. Additionally, this paper proposes new spectral calibration device named OCE (On-orbit Calibration Equipment) which consists of the visible and near infrared LEDs. The calibration accuracy by OCE compared with spectral lines of mercury and xenon has been evaluated. The OCE-BBM to central pixel of spatial direction can be calibrated with sufficient accuracy.
\end{abstract}

Key Words: Hyperspectral Sensor, Atmospheric Correction Error, On-orbit Calibration Equipment, LED

\section{Introduction}

The hyperspectral sensors have been a focus of attention in lots of application such as agriculture, environment and geology. The hyperspectral sensors acquire spectral information from objects with a high spectral resolution compared with multispectral sensors. Therefore, it enables to distinguish a targeted object with a high accuracy, and give us lots of important information. A typical image generated by hyperspectral sensor is shown in Fig. 1. The hyperspectral technology is very attractive not only to space business but also, academic applications. In general, the earth observation satellites have to provide the observational data which has high accuracy assurance. Additionally, the hyperspectral data includes not only the ground surface spectra, but also the effect of atmosphere. The hyperspectral data has to be corrected to provide the accurate information. But, the conditions such as contamination, radiation, high temperature and vibration environment affect the accuracy which was managed by pre-flight calibration. The hyperspectral data that have wavelength errors provide erroneous atmospheric correction with potential for erroneous classification of objects Therefore, we need on-orbit calibration technique being valid. The demonstrated spaceborne hyperspectral sensor to date was HYPERION $^{1)}$ from NASA (National Aeronautics and Space Administration) and CHRIS $^{2)}$ from ESA (European Space Agency). These sensors were calibrated by the Atmospheric Limb Corecction ${ }^{3)}$. On the other hand, the calibration process which used $\mathrm{O}_{2}$ and $\mathrm{H}_{2} \mathrm{O}$ absorptions are not enough accuracy that is required for hyperspectral sensor. This paper proposes LED (Light Emitting Diode) as on-board calibration subsystem. This paper first examined the effect of erroneous atmospheric correction on radiometric accuracy. Also we examined the effects of erroneous atmospheric correction on erroneous classification of the objects. And, this paper propose new on-board spectral calibration device which consists of LEDs to ensure high standard hyperspectral sensor.

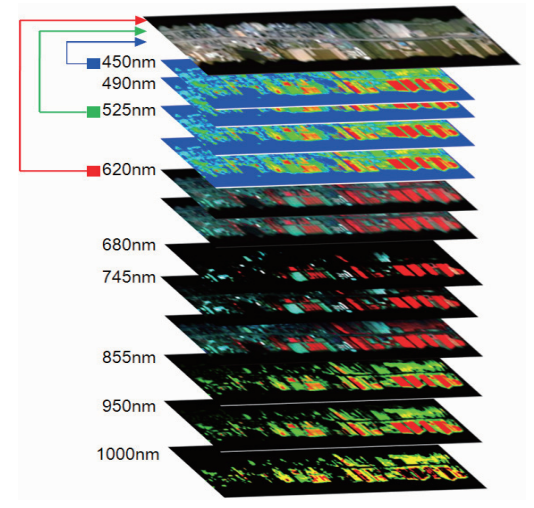

Fig. 1. Typical image generated by hyperspectral sensor.

\section{Space-Science Industries Program}

2.1. TAIKI with hyperspectral remote sensing mission

The Space-Science Industries Program has a goal which is building some space businesses. The program was kicked off in 2003 by the volunteer group that consists of students, researchers and engineers. In the program, we are planning to launch Micro-satellite as the demonstration for the space industries models. The earth observation micro-satellite "TAIKI" shown Fig. 2 is $50 \mathrm{~kg}$ satellite which has low-cost and small bus-subsystem for hyperspectral remote sensing. The bus-subsystem will be developed as manufactured products and keeps a lid on development cost to within 1 million dollars. In 2006, we also have successfully launched pico-satellite "HIT-SAT" which has mission of demonstrated bus-subsystem using lots of COTS (Commercial-Off the Shelf) components for TAIKI. This satellite is characterized by a spaceborne hyperspectral sensor "HSC-III",4). The mission objectives of TAIKI are summarized in the following: 
1) To provide hyperspectral image for agricultural remote sensing, 2) To acquire visualization of the effect of climate change on plant distribution.

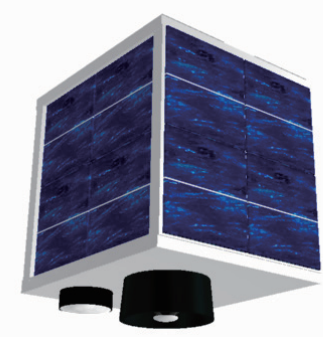

Fig. 2. The artist's view of TAIKI spacecraft.

\subsection{HSC-III}

The development of the HSC-III was started in January 2008 based on the optical design of the HSC1700 which was made as spin-off products of spaceborne hyperspectral sensor $^{5)}$. The key requirements of the HSC-III instrument call for a GSD (Ground Sample Distance) of $30 \mathrm{~m}$, a spectral range of 400-1000 nm containing 61 bands of $10 \mathrm{~nm}$ resolution, SNR (Signal to Noise Ration) of $>300$, and an instrument mass of $\leq 10 \mathrm{~kg}$. The imaging method adopted push-bloom technique. The overall objectives are to demonstrate the technology in orbit and to use the imagery in support of practical applications such as agricultural monitoring. HSC-III mainly consists of the telescope, the imaging spectrometer, the electrical system and inertial reference unit. The telescope has a pupil diameter of $0.2 \mathrm{~m}$, and has two mirror configuration of Ritchey-Chretien type. The spectrometer has the transmitting grating with the slit and relay-lens unit, and array sensor using back-illuminated CMOS image sensor. HSC-III optical design is presented in Fig. 3. The requirement specifications are summarized in Table 1 .

Table 1. HSC-III key requirements.

\begin{tabular}{|l|l|}
\hline Parameter & Requirements \\
\hline \hline Size & $>78 \times 24 \times 24 \mathrm{~cm}^{3}$ \\
\hline Weight & $10 \mathrm{~kg}$ \\
\hline Power consumption & $10 \mathrm{~W}$ \\
\hline Imaging type & Push-bloom method \\
\hline $\begin{array}{l}\text { Ground Sampling Distance } \\
\text { (GSD) }\end{array}$ & $30 \mathrm{~m}$ \\
\hline Swath width & $20.6 \mathrm{~km}$ \\
\hline FOV & $1.8 \mathrm{deg}$ \\
\hline Telescope aperture & $20 \mathrm{~cm}$ \\
\hline Wavelength range & $400-1000 \mathrm{~nm}(61 \mathrm{bands})$ \\
\hline Spectral resolution & $10 \mathrm{~nm}$ \\
\hline Maximum input & Albedo 70\% \\
\hline SNR & $>300 @ 620 \mathrm{~nm}$ \\
& $>200 @ 400-1000 \mathrm{~nm}$ \\
\hline Digitization & $10 \mathrm{bit}$ \\
\hline Mass memory & $30 \mathrm{~GB}$ \\
\hline
\end{tabular}

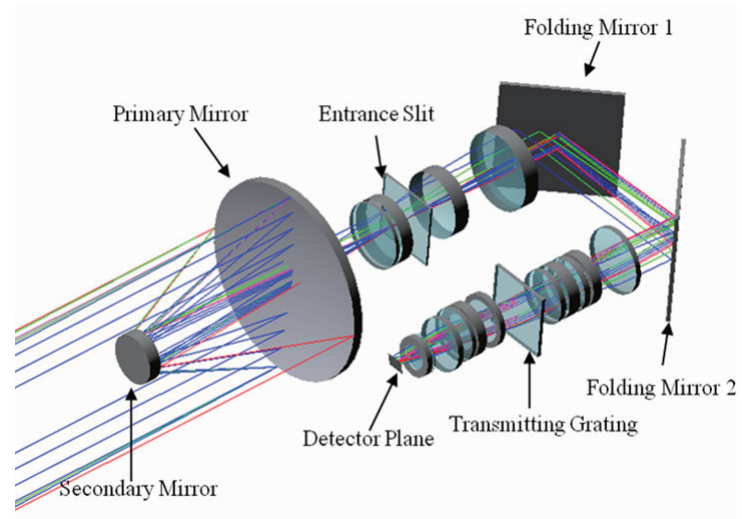

Fig. 3. HSC-III optical instrument.

A BBM (Breadboard Model) of the HSC-III optics instrument was developed in 2008. The BBM spectrometer instrument is shown in Fig. 4. It includes the entrance slit, the transmitting grating and relay-lens unit. The entrance light from the slit is dispersed by the grating onto the detector which is an area array sensor. The slit is $15 \mathrm{~mm}$ long by $15 \mu \mathrm{m}$ wide. The transmitting grating has an advantage which enables dispersion of entrance light into a spectrum of constant angle, so a spectral band width will become constant. The grating is $25 \mathrm{~mm}$ long by $25 \mathrm{~mm}$ wide, 300 lines $/ \mathrm{mm}$ grating frequency and approximately $75 \%$ maximum efficiency, and the grating made of B270 glass. The spectral band width was approximately $10 \mathrm{~nm}$. It has sufficient value compared with requirement.

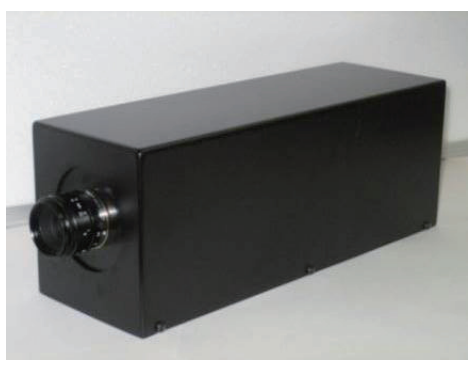

Fig. 4. Breadboard model of spectrometer.

\section{Required Spectral Accuracy for Hyperspectral}

\subsection{Radiometric simulation under the wavelength error}

Radiometric simulator between satellite and ground that has purpose of determination of the requirement for spectral calibration accuracy has been developed. This paper examined the effect of atmospheric correction on that the sensors have wavelength error. First, solar radiance spectrum (Kurucz, 2005) model $^{6)}$ that added spectral characteristics from objects is calculated in this simulator. It included the spectral characteristics from spectral library by using ASTER spectral library ${ }^{7}$. The reflected radiance model is converted to 10 bit digital data and 61 spectral bands. Figure 5 shows the reflected radiance that is targeted at the albedo $30 \%$, grasses and soils. The predicted spectral radiance reflection added the wavelength bias error of $1.5 \mathrm{~nm}$ to $0.25 \mathrm{~nm}$ is simulated that 
shown in Fig. 6 to Fig. 8. These results strikingly revealed that the wavelength error has a significant effect on radiometric error of $\mathrm{H}_{2} \mathrm{O}$ band $(760 \mathrm{~nm})$ and $\mathrm{O}_{2}$ band $(930 \mathrm{~nm}) . \mathrm{H}_{2} \mathrm{O}$ band is usually used for estimation of water contents, and $\mathrm{O}_{2}$ band is used for estimation of vegetation indexes. In consequence, it is assumed that hyperspectral data that has wavelength error affect the practical application for agriculture.

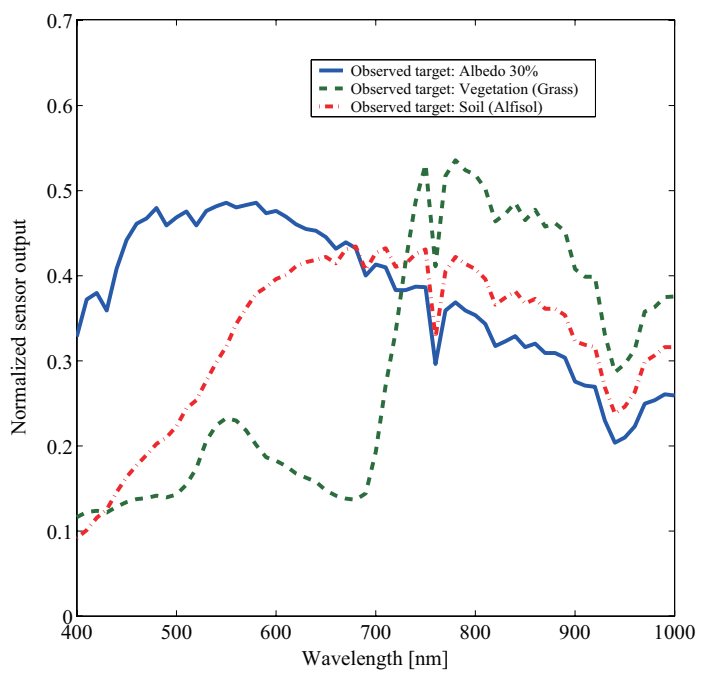

Fig. 5. Spectral radiance reflection.

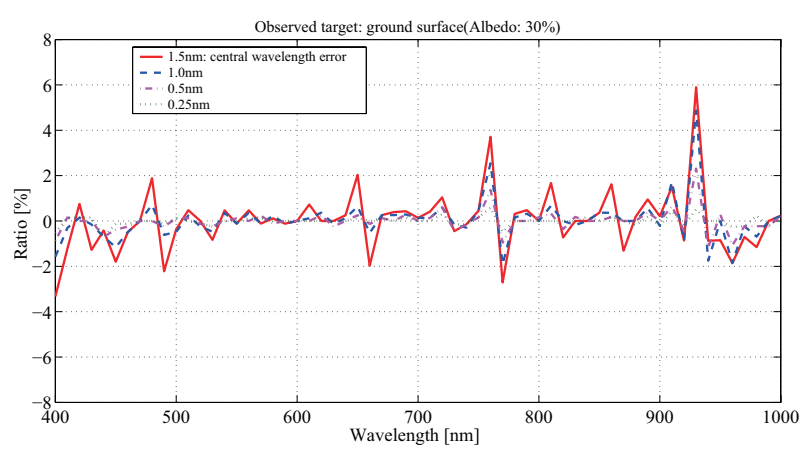

Fig. 6. Relative error of spectral radiance reflection (Albedo 30\%).

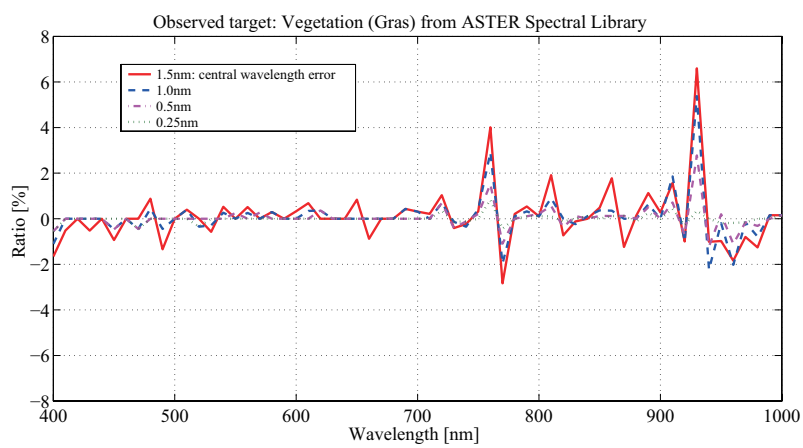

Fig. 7. Relative error of spectral radiance reflection (Grasses).

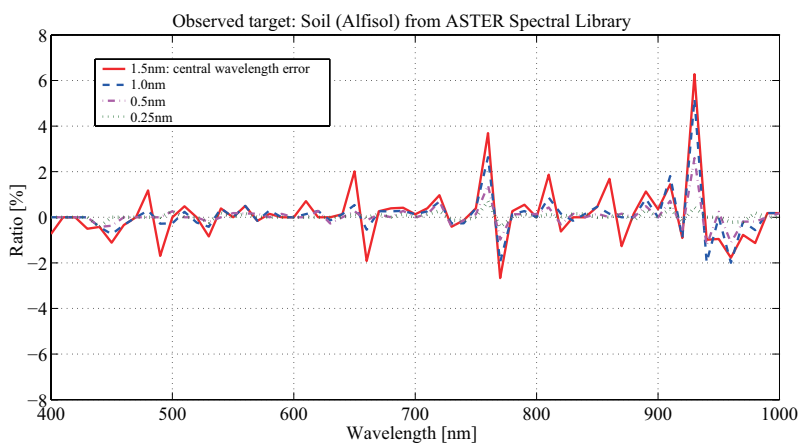

Fig. 8. Relative error of spectral radiance reflection (Soils).

\subsection{Effects of the erroneous classification}

The classification simulator by using hyperspectral data has been modeled under the condition that each spectral band has wavelength error. The acceptable accuracy for agriculture is decided in this simulator. We have chosen three pasture plants consist of Alfalfa (leguminosae), Orchard (grass) and Timothy (grass) as classification target. The procedure of classification is shown in Fig.9. The targets that reduced dimension by $2^{\text {nd }}$ derivative spectrum are classified by using maximum likehood method $^{8)}$. Fig.10 shows the classification example that was provided by using HSC1700. The result is summarized in Table 2. The results show no correlation between the wavelength error and the classification accuracy of Alfalfa and Orchard. However, if the data has more than $0.5 \mathrm{~nm}$ of wavelength error, the result shows that classification accuracy which is targeted at Timothy provides less than $70 \%$. Fig. 11 shows that $\mathrm{O}_{2}$ absorption band $(760 \mathrm{~nm})$ on the spectral curve of Timothy is most affect by the erroneous of atmospheric correction. According to simulation of atmospheric correction, $\mathrm{H}_{2} \mathrm{O}$ and $\mathrm{O}_{2}$ band are strikingly affected by the wavelength error. The erroneous classification can be estimated to be effect of wavelength error that each spectral band have. As a result, it has been decided that the spectral calibration accuracy is more than $0.25 \mathrm{~nm}$.

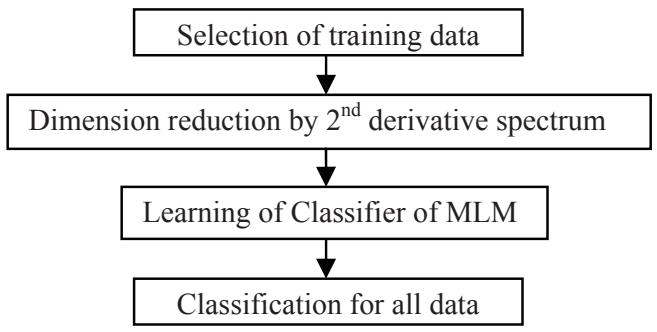

Fig. 9. Overview of the procedure of classification.
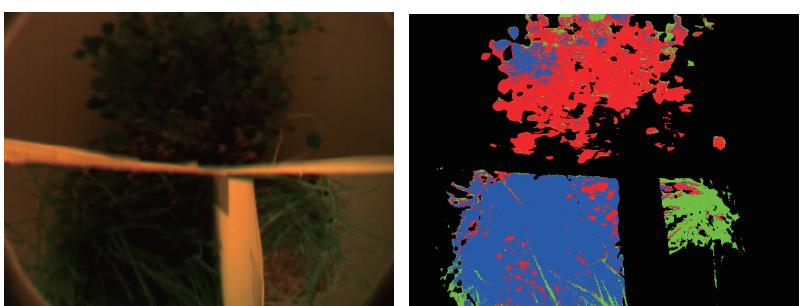

Fig. 10. Classification example by using hyperspectral data. 


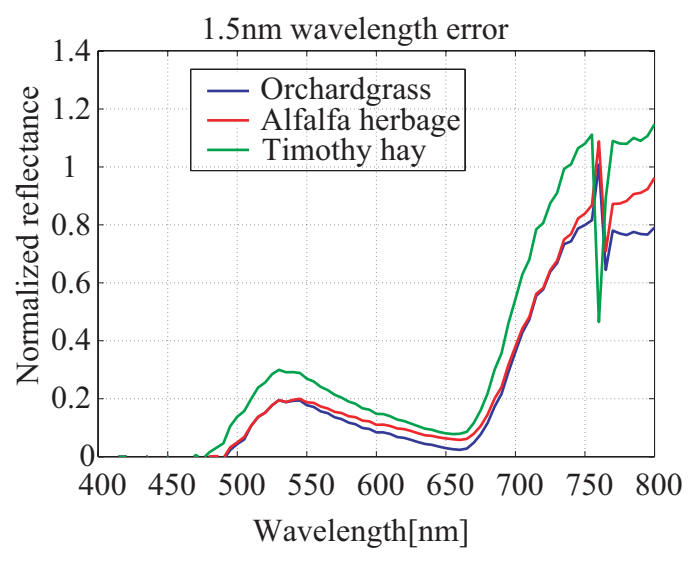

Fig. 11. Spectral characteristics under the wavelength error.

Table 2. Classification accuracy of pasture plants on the simulation.
\begin{tabular}{|l|c|c|c|c|c|}
\hline \multirow{2}{*}{ Object } & \multicolumn{5}{|c|}{ Wavelength error [nm] } \\
\cline { 2 - 6 } & 0.00 & 0.25 & 0.50 & 1.00 & 1.50 \\
\hline Alfalfa & $81.3 \%$ & $83.1 \%$ & $73.5 \%$ & $74.9 \%$ & $93.4 \%$ \\
\hline Orchard & $86.9 \%$ & $88.5 \%$ & $89.7 \%$ & $90.4 \%$ & $90.4 \%$ \\
\hline Timothy & $84.8 \%$ & $83.1 \%$ & $68.5 \%$ & $65.4 \%$ & $60.9 \%$ \\
\hline Total & $84.4 \%$ & $84.9 \%$ & $77.8 \%$ & $81.7 \%$ & $82.8 \%$ \\
\hline
\end{tabular}

\section{On-orbit Calibration Equipment}

\subsection{Proposed overview}

It is crucial to consider the on-orbit spectral calibration technique of hyperspectral sensor as suggested above. HYPERION and CHRIS have been calibrated by Atmospheric Limb Correction ${ }^{1)}$. On the other hand, these calibration processes are not enough accuracy that is required for hyperspectral sensor. To ensure high standard hyperspectral sensor should have subsystem for on-board calibration. The OCE (On-orbit Calibration Equipment) which is characterized by LED array has been proposed by our research. HSC-III will be equipped with the on-board OCE shown in Fig. 12. For spectral and relative radiometric calibration provides visible LEDs, near-infrared LEDs and diffused panel in front of the spectrometer entrance slit. The diffused panel provides three positions for earth observation mode, radiometric calibration, spectral calibration mode, and LED array monitoring mode. In addition the locking mechanism is necessary in order to inhibit vibration by launch vehicle. The OCE is equipped with monitoring photodiodes for radiometric calibration of each LED. The wavelength shift of the especially near-infrared LED is approximately $0.2 \mathrm{~nm} / \mathrm{K}$. In addition the required spectral accuracy is less than $0.25 \mathrm{~nm}$. As a consequence the absolute temperature of the LED array must be kept within $\pm 0.5 \mathrm{~K}$. The LED array will be equipped with TEC (Thermocooler) unit which included thermo resistor and thermoelectric cooler (such as Peltier device) in order to spectral calibration stability.

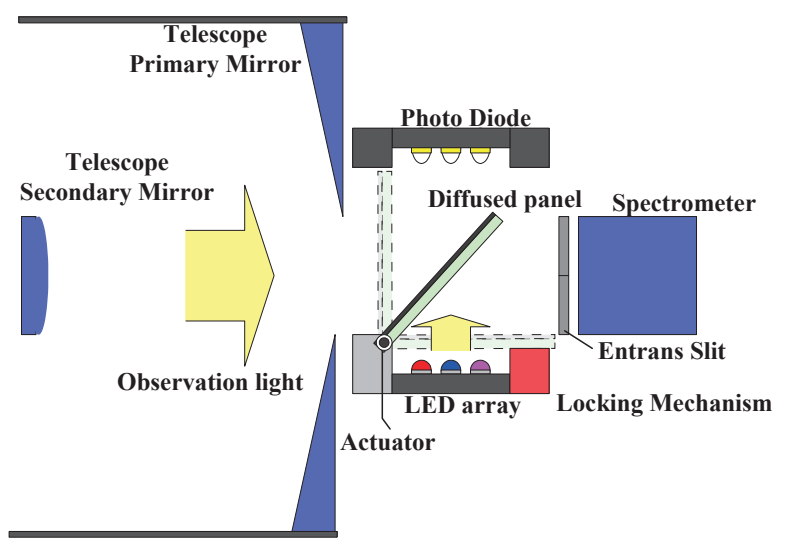

Fig. 12. On-orbit Calibration Equipment.

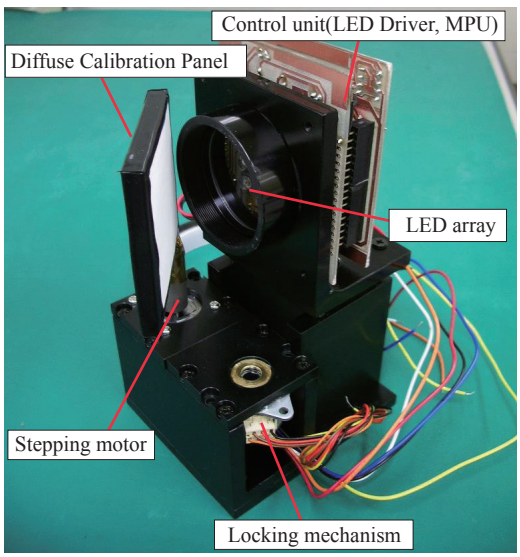

Fig. 13. BBM of On-orbit Calibration Equipment overview.

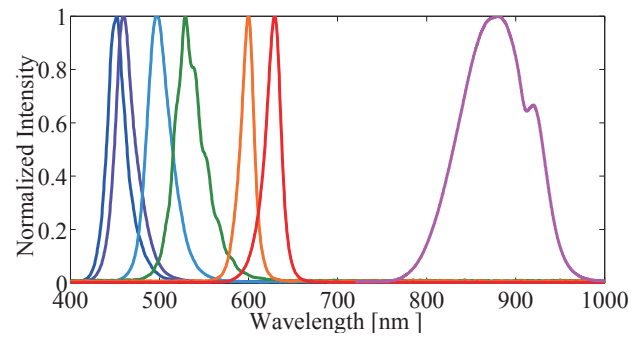

Fig. 14. LED array characteristics.

\begin{tabular}{ll}
\multicolumn{2}{c}{ Table 3. BBM of OCE parameters. } \\
\hline Parameter & Value \\
\hline \hline Size & $56 \times 90 \times 122 \mathrm{~mm}^{3}$ \\
\hline Power consumption & $3 \mathrm{~W}($ Average $)$ \\
\hline Weight & Approx. $1 \mathrm{~kg}$ \\
\hline Controller & $\begin{array}{l}\text { 32bit RISC microprocessor } \\
\text { (SH7125) }\end{array}$ \\
\hline Diffused panel & Material: Spectralon \\
& Size: $50 \times 50 \mathrm{~mm}^{2}$ \\
\hline
\end{tabular}

\subsection{Breadboard model of OCE}

This year the breadboard model of the OCE (OCE-BBM) which mainly consists LED array and diffused panel was developed. Fig. 13 shows the overview of the OCE-BBM. It 
features 6 high-intensity visible LEDs and a near-infrared LED. The LEDs characteristics are shown in Fig. 14. In addition the OCE is equipped with a high intensity white LED for radiometric calibration. To diffuse the calibration light source the SPECTRALON panel is used. The locking mechanism adopted the linear stepping motor. The OCE-BBM was equipped with a stepping motor for mode selection, but redundant actuator and monitoring photodiodes are still not equipped. The specifications are summarized in Table 3.

\subsection{Experiment of the spectral calibration}

This section describes the experiment of the on-board spectral calibration by using OCE-BBM. The equipment layout is shown in Fig. 15. By using OCE calibrated spectrum of the HSC-III spectrometer instrument (BBM). First of spectral calibration step calculates the relation between center wavelength of each LED and pixel number of targeted spectrometer by using centroid method. And by the least-square method calculates cubic polynomial which is spectral calibration curve. Table 4 summarizes the relation of center wavelength and pixel number of spectrometer. The center wavelength of each LED was measured by other spectrometer which features spectral resolution of $0.1 \mathrm{~nm}$ and wavelength range of 350 to $1150 \mathrm{~nm}$.

The calibration accuracy by using OCE-BBM has been evaluated. For comparing spectral accuracy used mercury lamp and xenon lamp (Spectral line: 404.66nm, 435.84nm, $546.07 \mathrm{~nm}, 579.07 \mathrm{~nm}$ and $883.79 \mathrm{~nm}$ ). The OCE-BBM to central pixel of spatial direction can be calibrated with sufficient accuracy as can be seen from Fig. 16. The each spectral residuals less than $0.03 \mathrm{~nm}$ was depicted in the figure.

The calibration results of far left pixel and far right pixel are shown in Fig. 17 and Fig. 18. The infrared spectrum has large residuals as can be seen from both figures. Especially the far right pixel has residuals of more than $0.3 \mathrm{~nm}$. Fig. 19 shows the residual relation of pixel number of spatial direction. The right pixel has larger residual, which is confirmed from the figure. We estimated the primary reason for this is effect of field curvature because the farther pixels have larger curvatures. In addition we estimated the other reason for this is effect of reduced receiving power of pixel end. However, the residuals have less than $0.2 \mathrm{~nm}$ (requirement) under the conditions that spatial pixels are within 600 pixels with sufficient accuracy.

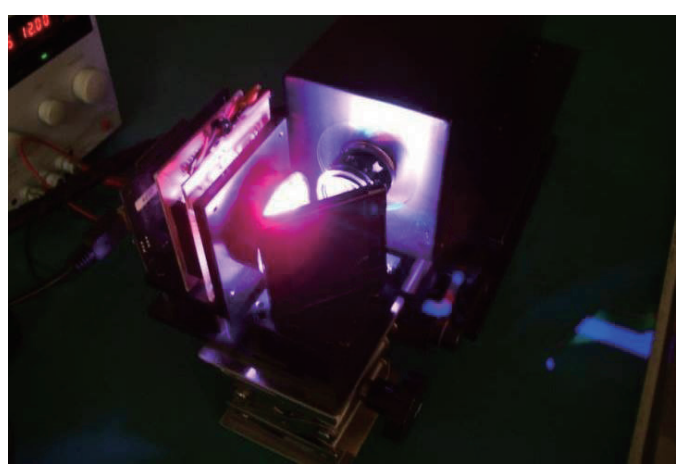

Fig. 15. Overview of the spectral calibration by OCE.
Table 4. Relation of center wavelength and pixel number.

\begin{tabular}{|c|c|}
\hline Center wavelength [nm] & Pixel number \\
\hline 449.44 & 102.32 \\
\hline 458.66 & 107.45 \\
\hline 497.01 & 129.36 \\
\hline 529.42 & 147.54 \\
\hline 599.59 & 186.87 \\
\hline 627.56 & 202.71 \\
\hline 874.74 & 340.64 \\
\hline
\end{tabular}
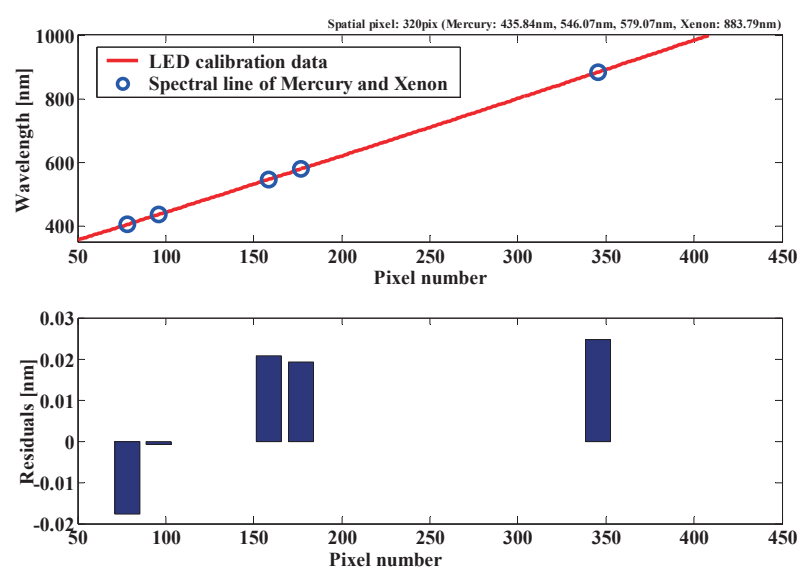

Fig. 16. Residual compared with each spectral line (Central pixel).
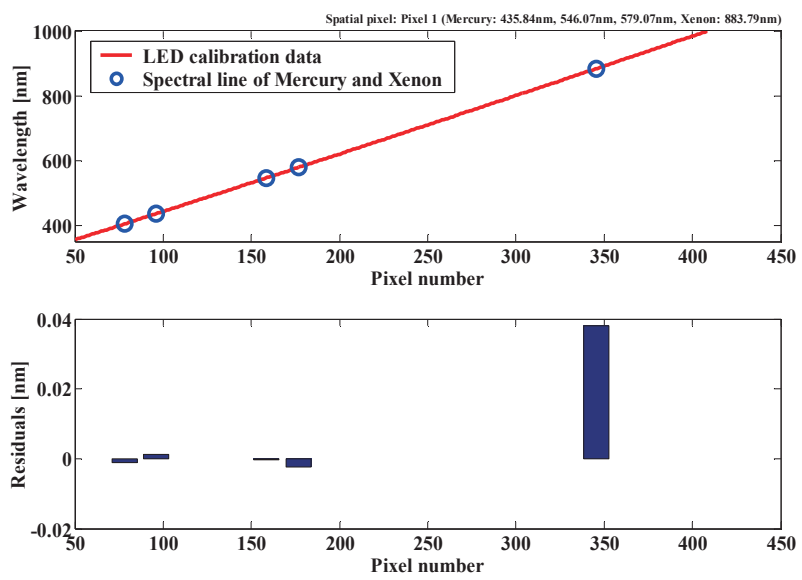

Fig. 17. Residual compared with each spectral line (Far left pixel).
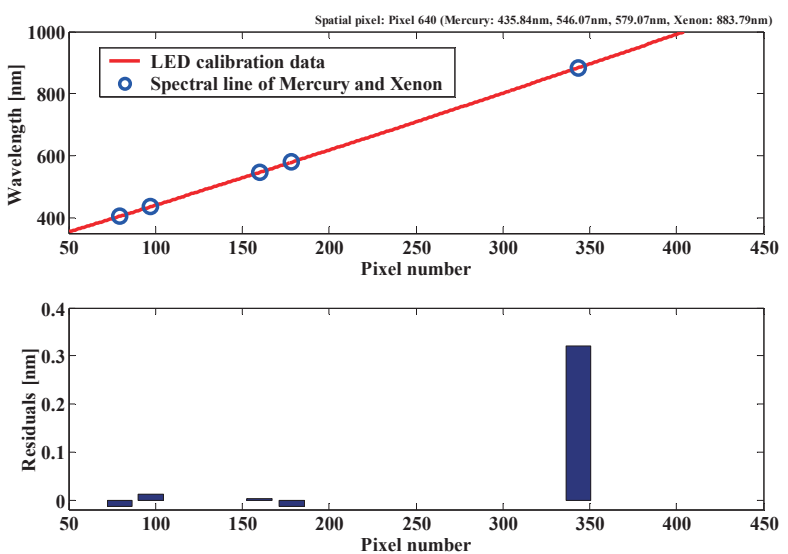

Fig. 18. Residual compared with each spectral line (Far right pixel). 


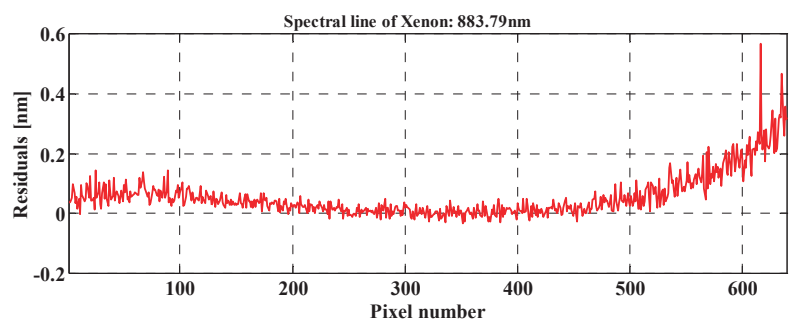

Fig. 19. Residual compared with infrared spectral line of Xenon.

\section{Conclusion}

This paper described the on-orbit calibration for the hyperspectral sensor. It was determined that required spectral calibration accuracy is more than $0.2 \mathrm{~nm}$ by using simulator which consists of radiometric model and classification algorithm. As a consequence, we proposed the OCE which is characterized by LED array. The OCE will be onboard instrument HSC-III. The LED array comprises 6 high intensity visible LEDs and a near-infrared LED. In this paper OCE-BBM has been developed and evaluated. For comparing spectral accuracy used mercury lamp and xenon lamp. The OCE-BBM to central pixel of spatial direction can be calibrated with sufficient accuracy. On the other hand, especially the infrared spectral bands of far right pixel have residuals of more than $0.3 \mathrm{~nm}$. As the solutions for this issue, the instrument should be equipped with additionally infrared LED for increasing the calibrated plots of infrared spectral bands. In conclusion, the results of this development study suggest that LED array may be good calibration light for the hyperspectral sensors. However, the calibration accuracy of the OCE should be evaluated statistically. The data in the paper were not sufficiently detailed to allow evaluation of temperature and vibration variations. In the future plan, the OCE-BBM must be equipped with Thermocooler unit and monitoring photodiodes, and we are planning to evaluate by the vibration test and temperature test.

\section{References}

1) Pearlman, J., Carman, S., Segal, C., Jarecke, P., Clancy, P. and Browne, W.: "Overview of the Hyperion Imaging Spectrometer for the NASA EO-1 Mission", Geoscience and Remote Sensing Symposium, 7 (2001), pp. 3036-3038.

2) Barnsley, M., Settle, J., Cutter, A., Lobb, R. and Teston, F.: "The PROBA/CHRIS Mission: A Low-Cost Smallsat for Hyperspectral, Multi-Angle, Observations of the Earth Surface and Atmosphere", IEEE Transactions on Geoscience and Remote Sensing, 42 (2004), issue 7, pp. 1512-1520.

3) Barry, P., Shepanski, J. and Segal, C.: "Hyperion on-orbit validation of spectral calibration using atmospheric lines and an on-board system”, Proc. SPIE 4480 (2002), pp. 231-235.

4) Aoyanagi, Y., Satori, S., Totani, T., Yasunaka, T., Nakamura, A. and Takeuchi, Y.: "Development of Spaceborne Small Hyperspectral sensor HSC-III for Micro Satellite", Small Satellites for Earth Observation, Digest of the $7^{\text {th }}$ International Symposium of the International Academy of Astronautics, 2009, pp. 183-186.

5) Satori, S., Aoyanagi, Y., Nishizato, S., Totani, T., Yasunaka, T., Nakamura, A. and Takeuchi, Y.: "Development of Hyperspectral sensor for Hokkaido Satellite 'TAIKI'", 59 ${ }^{\text {th }}$ International Astronautical Congress, Glasgow, Scotland, 2008.

6) KITT Peak Solar Flux Atlas, 2005, http://kurucz.harvard.edu/

7) ASTER Spectral Library, http://speclib.jpl.nasa.gov/

8) Ishimura, K., Wada, M., Inagaki, Y., Satori, S. and Nakazawa, M.: "Classification of Plants by Using Hyperspectral Data", Journal of the Visualization Society of Japan, 28 (2008), pp.145-146. 